\title{
Análisis de Decisiones en la Selección de Proveedores de Tecnologías de la Información: Una Revisión Sistemática ${ }^{1}$
}

\author{
Lucas Grossi $^{1}$, Jose A. Calvo-Manzano ${ }^{1}$ \\ lgcgrossi@gmail.com,joseantonio.calvomanzano@upm.es \\ ${ }^{1}$ Facultad de Informática, Universidad Politécnica de Madrid, 28660, Boadilla del Monte, \\ España.
}

\begin{abstract}
Resumen: En este artículo se aplica un protocolo de revisión sistemática de Ingeniería de Software para la decisión en la selección de proveedores de Tecnologías de la Información (TI). El objetivo es la búsqueda de documentos relacionados con la toma de decisión en la selección de proveedores de TI. Además, la revisión sistemática se centra en identificar las iniciativas y los informes de la toma de decisión en la selección de proveedores de TI. Los resultados obtenidos demuestran que existe una necesidad de más estudios y definen 16 criterios de evaluación para utilizarse en la selección de proveedores de TI.
\end{abstract}

Palabras clave: Selección de Proveedores; Análisis de Decisiones; Outsourcing; Revisión Sistemática.

\begin{abstract}
This paper applies a Systematic Review protocol for Software Engineering to IT outsourcing supplier selection decision. The objective is to search for papers related to IT outsourcing supplier selection decision. Furthermore, the systematic review is focused in order to identify initiatives and reports of IT outsourcing supplier selection decision proposals. Results obtained show that there is a need for more in-depth studies.
\end{abstract}

Keywords: Supplier Selection, Decision Analysis, Outsourcing, Systematic Review.

\section{Introducción}

Las actividades de negocios de hoy dependen en gran medida del apoyo de las Tecnologías de la Información (TI), por lo que su mantenimiento es un proceso crítico

${ }^{1}$ Este trabajo ha sido patrocinado por la Fundación everis y la Universidad Politécnica de Madrid a través de la Cátedra de Mejora de Procesos de Software para España y América Latina. 
y debe realizarse con la mayor calidad posible. El problema de la selección de proveedores de TI requiere conocimientos específicos de la organización y de la técnica para lograr la deseada calidad de TI, y puede ser una tarea desalentadora para los gerentes que no están seguros acerca de sus múltiples implicaciones.

En los últimos años, el outsourcing de TI ha cobrado una gran importancia en el mercado y continúa creciendo cada año. Outsourcing como concepto ganó la aceptación común en la década de los 80 y, hoy en día, todavía se utiliza para describir "una relación contractual con un proveedor de servicios especializados para el trabajo tradicionalmente hecho en casa" (Corbet \& Associates, 2002).

Analistas de tendencias, como Morgan \& Chambers (Lee, 2009) e IDC (Gellings, 2007), predicen cifras de crecimiento anual de aproximadamente el 10\%. IDC estima que los clientes en EE.UU. aumentarán su gasto en outsourcing a partir de 2011 cuando la economía reviva y espera que el gasto de estos servicios llegue a un total estimado de $\$ 57$ mil millones en 2014 (Dialani, 2010). Aarkstore (Aarkstore, 2010) prevé que el gasto anual en outsourcing superará los $\$ 700$ mil millones a nivel mundial en el año 2013. Huai (Huai, 2007) demostró que el mercado de outsourcing de SI (sistemas de información) creció rápidamente durante los últimos 20 años con estimaciones de que los ingresos de outsourcing crecerán un $6,2 \%$.

Aunque la popularidad del outsourcing de TI creció en las últimas dos décadas, muchos acuerdos de subcontratación no han continuado. Un estudio de (Dorothy \& Dwayne, 2006) encontró que aproximadamente un tercio de las empresas estudiadas habían cancelado los contratos de outsourcing. También, de acuerdo con un estudio de (CMMI, 2010), del 20 al 25 por ciento de los proyectos de adquisición de TI de gran tamaño fracasan dentro de dos años y el 50 por ciento fracasan dentro de cinco años. Los factores que contribuyen al fracaso del proyecto son: la mala gestión, la mala definición de requisitos, la falta de evaluaciones comprensivas, la selección de proveedores y la contratación inadecuada, los insuficientes procedimientos tecnológicos de selección, y los cambios de requisitos no controlados. La mayoría de los fracasos de proyectos se podrían evitar si el adquirente aprendiera a: comprender los problemas de decisión, hacer un mejor análisis de decisiones y usar el buen juicio (CMMI, 2010). (Gill, Grewal, \& Sareen, 2008) también indican que la mala decisión en la selección del proveedor de TI es una de las razones fundamentales que causa el alto fracaso del outsourcing de TI. Por lo tanto, el proceso científico de toma de decisión en la selección de proveedores de outsourcing de TI es muy importante para aumentar la tasa de éxito del outsourcing.

El trabajo presentado en este documento muestra los resultados de una revisión sistemática sobre la decisión en la selección de proveedores de TI en las áreas de tecnologías de la información y sistemas de información.

\section{Revisión Sistemática sobre Análisis de Decisiones en la Selección de Proveedores de TI}

Antes del siglo XX no había métodos para integrar los resultados de la investigación. En 1904, Pearson calculó la media de los resultados de la correlación entre la inoculación de la fiebre tifoidea y la mortalidad. Entonces la revisión sistemática 
comenzó a formalizarse y a finales de los años 8o, la revisión sistemática logra legitimidad como campo de investigación (Aydin \& Bakker, 2008; Basili, Craze, Jino, Mendonca, \& Shull, 2007).

La búsqueda de temas relacionados con la toma de decisión en la selección de proveedores de outsourcing de TI se basó en el trabajo de (Biolchini, Mian, Natali, \& Travassos, 2005), que propone un protocolo de revisión sistemática sobre las directrices propuestas por (El-Emam et al., 2001), y en la forma de extraer información de documentos de ingeniería de software, desarrollado por (Basili, et al., 2007) y otras revisiones sistemáticas de índole similar desarrolladas por (Garcia, Piattini, \& Pino, 2008), (Calvo Manzano, Cuevas, Gasca, \& San Feliu, 2009) y (Calvo-Manzano, Cuevas, Grossi, Mejia, \& San Feliu, 2009). La revisión sistemática desarrollada durante este trabajo siguió el protocolo establecido por los autores indicados anteriormente. En las siguientes secciones se presenta la revisión sistemática realizada sobre la toma de decisión en la selección de proveedores de TI.

\subsection{Formulación de la pregunta}

Esta sección tiene por objetivo definir la sintaxis de la pregunta de investigación (el contexto/problema en el que se aplica la revisión y la pregunta que el estudio debe responder) y su especificidad semántica (alcance de la pregunta) descrito por los elementos restantes de esta sección - intervención, control, efecto, medida de resultado, población y aplicación (Basili, et al., 2007). A continuación, se describe cada uno de ellos para la toma de decisión en la selección de proveedores de TI.

- Problema: el proceso de análisis de decisiones es muy importante en cualquier selección de proveedores de TI. El éxito del proyecto se relaciona con una buena decisión en la selección de proveedores. Por ello, se necesita desarrollar un modelo de toma de decisión en la selección de proveedores de TI.

- Pregunta: ¿cuáles son las iniciativas o propuestas desarrolladas para la toma de decisión en la selección de proveedores de TI?

- Intervención: el desarrollo actual de la toma de decisión en la selección de proveedores de TI.

- Control: no hay datos iniciales para esta revisión sistemática.

- Efecto: iniciativas y propuestas relacionadas con la toma de decisión en la selección de proveedores de TI.

- Medida de resultados: número de propuestas identificadas.

- Población: publicaciones relacionadas con la toma de decisión en la selección de proveedores de TI.

- Aplicación: cualquier empresa que tiene que tomar decisiones en la selección de proveedores de TI. Los investigadores que trabajan con la toma de decisión en la selección de proveedores de TI.

\subsection{Selección de Fuentes}

El objetivo de esta sección es seleccionar las fuentes donde se llevará a cabo la búsqueda de estudios primarios (Basili, et al., 2007). Para llevar a cabo la selección, se propone a abordar las siguientes cuestiones (secciones). 


\subsubsection{Definición de los Criterios de Selección de Fuentes y Lenguajes de Estudio}

- Criterios de selección de fuentes: mecanismos de búsqueda con palabras clave y sitios sugeridos por expertos, artículos recomendados por otros expertos y artículos disponibles en los sitios Web.

- $\quad$ Lenguaje de estudio: inglés.

\subsubsection{Identificación de Fuentes}

- Método de búsqueda de fuentes: la identificación de las fuentes se ha basado en el criterio de expertos en nuestra área de investigación. Estas fuentes incluyen revistas como: European Journal of Operational Research, Information and Software Technology, Software: Practice and Experience, Software Process: Improvement and Practice, IEEE Software, Software Technology and Engineering Practice, talleres de investigación en computación e informes técnicos del Software Engineering Institute - SEI.

- Cadena de búsqueda: fueron extraídas palabras clave del conjunto de palabras definido en la "pregunta", combinando estas palabras clave con los operadores lógicos "AND" y "OR". Se han obtenido cuatro cadenas de búsqueda. Además, al analizar las referencias de los documentos iniciales encontrados, se han definido tres cadenas más de búsqueda. Estas cadenas de búsqueda se han adaptado para cada buscador de las fuentes. Las cadenas de búsqueda definidas han sido: 1) "decision analysis AND IT Acquisition; 2) "decision analysis" AND outsourcing; 3) "decision analysis AND resolution"; 4) "formal evaluation process" AND "information technology"; 5) decision AND (analysis OR resolution) AND "information technology"; 6) (supplier OR provider) AND selection AND outsourcing; 7) "decision analysis" AND (supplier OR provider) AND selection.

- Lista de fuentes: estas fuentes se han seleccionado teniendo en cuenta el método de búsqueda de fuentes definido. La lista de fuentes definidas ha sido: 1) Science@Direct; 2) Springer Link; 3) Computer Database - GALE; 4) ISI Web of Knowledge; 5) IEEE Computer Science Digital Library; 6) ACM Digital Library.

\subsection{Selección de Estudios}

En esta revisión sistemática se ha utilizado un procedimiento iterativo e incremental para la selección de estudios: a) iterativo, para agrupar todas las actividades que podrían repetirse durante el procedimiento, y b) incremental, ya que los estudios se abordan y se registran uno por uno, hasta la obtención de los resultados de la revisión sistemática (Garcia, et al., 2008). Se ha utilizado este procedimiento iterativo e incremental debido a su funcionalidad en otras revisiones sistemáticas. Esta sección describe el proceso y los criterios para la selección de los estudios y la evaluación. 


\subsubsection{Definición de los Criterios de Inclusión y Criterios de Exclusión de los Estudios, y Proceso de Selección}

Este elemento define como se han seleccionado los estudios (Basili, et al., 2007).

- Definición de los criterios de inclusión (CI) y de exclusión (CE) de los estudios: se presentan los criterios por los que los estudios serán evaluados para decidir si deben ser seleccionados o no en el contexto de la revisión sistemática (Basili, et al., 2007). Los criterios fueron definidos por los autores de este artículo teniendo en cuenta la propuesta de (El-Emam, et al., 2001) (véase la Tabla 1).

- Definición de los tipos de estudios: se tendrán en cuenta todos los estudios relacionados con la selección de proveedores de TI. Sin embargo, el mayor interés se centrará en estudios que muestren resultados en la toma de decisión en la selección de proveedores de TI, es decir, que definan un modelo o forma de estructurar el proceso de toma de decisiones en la selección de proveedores de TI.

- Procedimiento para la selección de estudios: teniendo en cuenta los criterios de selección, el título ha sido el primer criterio principal, pero, en algunos casos, no ha proporcionado suficiente información, por lo tanto la lectura del resumen de cada uno de ellos ha sido necesaria y, en otros casos ha sido necesaria una revisión del texto completo.

Tabla 1. Criterios de inclusión (CI) y exclusión (CE) de estudios

\begin{tabular}{ll}
\hline$\#$ & Criterios de inclusión (CI) y exclusión (CE) \\
\hline CI1 & $\begin{array}{l}\text { Incluir artículos cuyo título se relaciona con la toma de decisión en la selección de proveedores de } \\
\text { TI }\end{array}$ \\
\hline CI2 & $\begin{array}{l}\text { Incluir artículos que contienen palabras clave que coinciden con los definidos en la cadena de } \\
\text { búsqueda }\end{array}$ \\
\hline $\mathrm{CI}_{3}$ & Incluir artículos cuyo resumen está relacionado con el tema tratado \\
\hline $\mathrm{CI} 4$ & Incluir artículos después de la lectura parcial o completa \\
\hline $\mathrm{CE} 1$ & Excluir todos los artículos duplicados (aquellos seleccionados en varios motores de búsqueda) \\
\hline
\end{tabular}

\subsubsection{Ejecución de la Selección}

El proceso de selección de los estudios primarios y los resultados de su evaluación son presentados en esta sección (Basili, et al., 2007).

- Evaluación de la calidad de los estudios: aplicando los CIs y CE definidos anteriormente, fue posible encontrar los estudios primarios (véase Tabla 2). Esta tabla contiene los resultados obtenidos con las cadenas de búsqueda en cada motor de búsqueda, la cantidad de trabajos encontrados, los que no se repitieron, y los estudios relevantes y primarios encontrados. Aplicando los criterios de inclusión CI1, CI2 y CI3, sólo 72 de 1727 artículos encontrados fueron considerados relevantes. Aplicando el criterio CE1 para la exclusión de los estudios duplicados, 62 estudios fueron obtenidos (columna No Repetido). A partir de éstos, y aplicando el criterio CI4, sólo 52 estudios fueron seleccionados como primarios. 
Los estudios encontrados aquí son la base para la revisión sistemática y para el análisis que será presentado en las próximas secciones.

Tabla 2. Resultados derivados de cada fuente de búsqueda

\begin{tabular}{lllll}
\hline Fuente & Encontrado & Relevante & No Repetido & Primario (\%) \\
\hline ACM Portal & 656 & 16 & 14 & $9(17,3)$ \\
\hline IEEE Explore & 29 & 4 & 2 & $2(3,8)$ \\
\hline Computer Database Gale & 86 & 4 & 3 & $3(5,8)$ \\
\hline ISI Web of Knowledge & 484 & 31 & 31 & $31(59,6)$ \\
\hline Science@Direct & 245 & 11 & 8 & $3(5,8)$ \\
\hline Springer Link & 227 & 6 & 4 & $4(7,7)$ \\
\hline TOTAL & 1727 & 72 & 62 & $52(100)$ \\
\hline
\end{tabular}

\subsection{Extracción de la Información}

Una vez que se han seleccionado los estudios primarios, se puede iniciar el proceso de extraer la información útil. Este proceso puede comenzar justo después de la definición de los criterios de inclusión y de exclusión de la información.

Tabla 3. Criterios de inclusión (CI inf) y de exclusión (CE inf) de la información

\begin{tabular}{ll}
\hline$\#$ & Criterios \\
\hline CI1 inf & $\begin{array}{l}\text { Recopilar información sobre la tendencia de la organización relacionada con la toma de } \\
\text { decisión en la selección de proveedores de TI }\end{array}$ \\
\hline CI2 inf & Recopilar información sobre el área de trabajo (TI, SI, etc.) de los estudios \\
\hline CI3 inf & $\begin{array}{l}\text { Recopilar información sobre los modelos aplicados por los estudios para la toma decisión } \\
\text { en la selección de proveedores de TI }\end{array}$ \\
\hline CI4 inf & $\begin{array}{l}\text { Identificar metodologías, métodos y cualquier procedimiento propuestos en los estudios } \\
\text { para la toma de decisión en la selección de proveedores de TI }\end{array}$ \\
\hline CE1 inf & $\begin{array}{l}\text { Excluir las informaciones que no están relacionadas con los criterios de inclusión definidos } \\
\text { arriba }\end{array}$ \\
\hline
\end{tabular}

Para analizar la información de los estudios primarios, éstos fueron agrupados por carpetas relacionadas con la fuente de búsqueda donde fueron encontrados. Entonces, los estudios primarios de cada una de estas carpetas fueron copiados y pegados en una carpeta final para eliminar los repetidos. La información relevante de cada estudio primario fue destacada y luego extraída en un archivo separado, conteniendo el nombre del estudio primario y toda la información útil relacionada.

La ejecución de la extracción fue de la siguiente forma:

- Extracción de resultados objetivos: en busca de cualquier información relevante relacionada con la toma de decisión en la selección de proveedores de TI, los estudios seleccionados fueron leídos al completo. La información encontrada fue seleccionada y organizada en tablas que contienen: estudio consecutivo (número de documento secuencial), metodología de estudio (los comentarios de las principales ideas relacionadas con la metodología) y el 
resultado del estudio (datos e información de las conclusiones presentadas en cada estudio).

- Extracción de resultados subjetivos: para completar la información encontrada en la extracción objetiva, se añadieron dos datos más a la tabla: los datos sobre los autores (nombre completo e información de contacto disponible en los estudios) y notas adicionales (un campo específico para almacenar información general relacionada con el tema tratado en el estudio).

Siguiendo este procedimiento, la información obtenida fue organizada siguiendo los campos: número consecutivo, título del artículo, revista/conferencia, autores, tópico, tipo de estudio, fecha, país, metas (analizar, a fin de, con respecto a, desde el punto de vista de), método (nombre, tipo, valores posibles, procedimiento de recolección de datos), implementación (compañía, categoría, tamaño), resultados del estudio y otros temas. Este formato fue adaptado teniendo en cuenta otras revisiones sistemáticas, como la de (Garcia, et al., 2008) y la propuesta de extraer información del trabajo de (Basili, et al., 2007).

\section{Resumen de Resultados}

A continuación, hay que analizar la información extraída de los estudios primarios.

\subsection{Propuestas de los Estudios}

La mayoría de los estudios analizados están relacionados con el área de TI o SI, que son exactamente los temas que esta revisión sistemática se propuso encontrar. Estos estudios mencionan algunas ideas generales relacionadas con el proceso de la toma de decisiones utilizando modelos de criterios múltiples. La mayoría de las propuestas (71\%) están relacionadas con el diseño de un modelo específico para la toma de decisiones, mostrando cómo seleccionar la mejor alternativa según los criterios definidos. Algunas de las propuestas (23\%) analizaron el proceso de toma de decisiones, un 4\% se centró en el uso de las primitivas de decisión en el proceso de toma de decisiones y un estudio (el 2\%) presentó una lista de artículos relacionados con el asunto de toma de decisiones.

\subsection{Clasificación de los Estudios}

Los estudios primarios fueron seleccionados siguiendo las actividades definidas en la revisión sistemática y pudieron ser clasificados en áreas de interés. La clasificación muestra 5 tópicos diferentes relacionados con: Tecnologías de la Información, Sistemas de Información, Salud, Logística e Ingeniería Civil.

El 59\% de los estudios estaban relacionados con Tecnologías de la Información y el $27 \%$ con Sistemas de Información. Esta información muestra que la revisión sistemática logró uno de sus propósitos, ya que la mayoría de los estudios están relacionados con el área que estábamos buscando (Tecnologías de la Información y Sistemas de Información).

La mayoría de los estudios (37\%) utilizaron una mezcla de modelos (dos o tres) en el proceso de toma de decisiones. El Analytic Hierarchy Process (AHP) (Saaty, 1980) se utilizó en un 19\% de los estudios y PROMETHEE (Brans, Mareschall, \& Vincke, 1986) 
en un 10\%. Los modelos utilizados para hacer un análisis del proceso de decisión contribuyeron con un 8\% (Analytical Study) y un 12\% (Survey Methodology). Fuzzy Analytical Network Process (Mikhailov \& Singh, 2003) con (10\%) y Decision Primitives con (4\%) completan la clasificación de los estudios por modelo. Por lo tanto, es posible ver que la mayoría de los estudios utilizaron AHP o PROMETHEE como modelo de análisis de decisión, pero ninguno de ellos los utilizó juntos, tratando de captar las ventajas de cada modelo y evitar los inconvenientes.

\section{Análisis de los Resultados}

(Dickson, 1966) llevó a cabo una encuesta enviada por correo a cerca de 300 organizaciones comerciales, principalmente empresas de manufactura. A los gerentes de compras de estas empresas, se les pidió identificar los factores que eran importantes para la selección de proveedores. Sus hallazgos fueron divididos en dos categorías: las prácticas de selección de proveedores de las empresas y las prácticas de selección de proveedores de los individuos. Calidad, precio y entrega fueron identificados como los factores más críticos en el proceso de selección de proveedor. De acuerdo con sus resultados, el porcentaje de sistemas utilizando cada factor fue: Calidad (96,6\%), Precio (93,9\%), Entrega (93,9\%), Servicio (81,8\%), Capacidad Técnica (63,6\%), Fuerza Financiera (51,5\%), Localización Geográfica (42,4\%), Reputación (42,4\%), Acuerdos de Reciprocidad (15,1\%) y Otros Factores (12,1\%.)

Para estudiar las prácticas de selección de proveedores por los encargados de la toma de decisiones individuales, (Dickson, 1966) seleccionó cuatro escenarios únicos de compra que abarcan productos y situaciones muy diferentes, y una lista de 23 factores descritos en la literatura como importantes para el problema de la toma de decisión en la selección de proveedores. Cada entrevistado (gerente de compras) fue preguntado para considerar cada situación y evaluar la importancia de cada uno de los factores enumerados en una escala de o a 4 (4 representando importancia extrema y o indicando poca o ninguna importancia). Los factores definidos fueron (presentados en orden y con la media entre paréntesis):

- Importancia extrema: calidad $(3,5)$.

- Importancia considerable: entrega $(3,4)$, historia de desempeño $(3,0)$, garantías y políticas de reclamaciones $(2,9)$, instalaciones de producción y capacidad $(2,8)$, precio $(2,7)$, capacidad técnica $(2,5)$, y posición financiera $(2,5)$.

- Importancia media: cumplimiento de los procedimientos $(2,5)$, sistema de comunicación $(2,4)$, reputación y posición en la industria $(2,4)$, deseo de negocios $(2,3)$, administración y organización $(2,2)$, control de operación $(2,2)$, servicio de reparación $(2,2)$, actitud $(2,1)$, impresión $(2,0)$, capacidad de embalaje $(2,0)$, registro de relación laboral $(2,0)$, localización geográfica $(1,9)$, cantidad de trabajos anteriores $(1,6)$ y asistencia a la formación $(1,5)$.

- Pequeña importancia: acuerdos de reciprocidad $(0,6)$

Teniendo en cuenta la extensa naturaleza del estudio de (Dickson, 1966) y el enfoque estructurado adoptado, era apropiado extender sus resultados para incluir la investigación obtenida sobre el problema de toma de decisión en la selección de 
proveedores publicada hoy en día, de 1992 a 2010, donde 52 artículos, como se mostró en la Tabla 2 de la sección 2.3.2, fueron revisados y analizados. Es importante tener en cuenta que esta revisión se basa totalmente en la literatura académica, mientras que el estudio de (Dickson, 1966) se basó en una encuesta a los agentes de compras.

Tabla 4. Distribución de los Criterios de Evaluación por Artículos

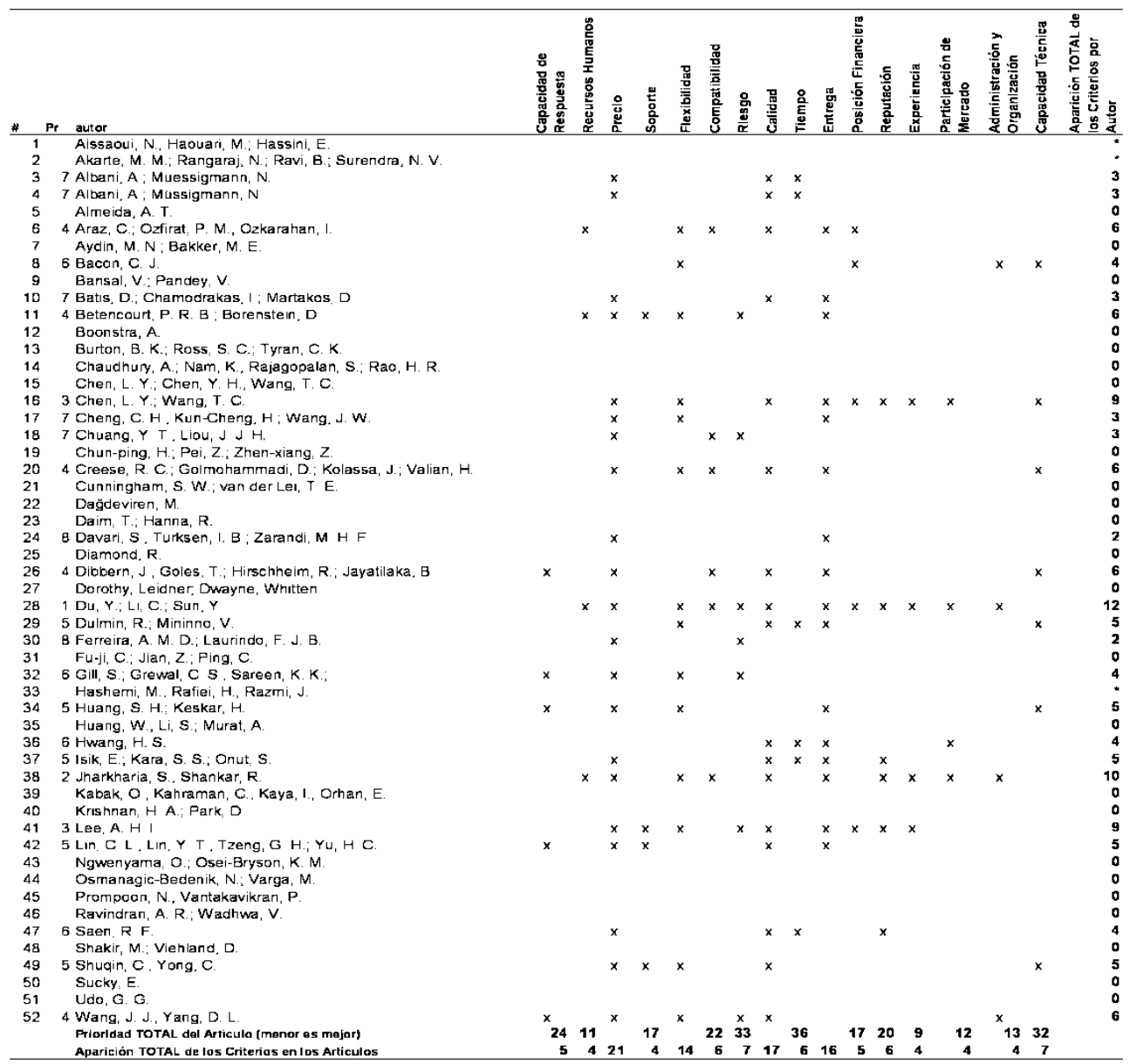

* mencionan la lista de Dickson.

La Tabla 4 clasifica a todos los estudios y los criterios de evaluación encontrados en cada uno de ellos. La primera columna es solamente un número de identificación del estudio, y la segunda columna muestra la prioridad del estudio (Pr) (donde los artículos con más criterios definidos fueron considerados los más prioritarios). Artículos con mismo número de criterios definidos tienen la misma prioridad, por eso los números repetidos en la segunda columna de la Tabla4 (por ejemplo, artículos 16 y 41, con prioridad 2), y artículos sin criterios definidos no tienen ninguna prioridad, por eso algunos artículos no tienen un número asignado (por ejemplo, artículos 1 y 2). La tercera columna lista los autores de los artículos encontrados en la revisión sistemática 
y las demás columnas contienen los nombres de los criterios definidos en este artículo, con excepción de la última columna (aparición total de los criterios por autor) que será explicada más adelante.

Una "x" en la Tabla 4, debajo de las columnas de los criterios, confirma el reconocimiento de un criterio en la investigación de selección de proveedores publicada en el estudio. Los nombres de los criterios se basaron en la lista de (Dickson, 1966) y en los nombres encontrados en los documentos analizados y, cuando dos criterios tienen una misma definición pero nombres diferentes, los autores de este trabajo decidieron poner el mejor nombre (según su criterio). Es importante mencionar que sólo fueron seleccionados los criterios que aparecieron en cuatro o más trabajos, es decir, otros criterios que fueron encontrados en el análisis de los documentos, pero que no aparecen en tantos estudios, no han sido incluidos.

En la Tabla 4 algunos cálculos y análisis fueron realizados para obtener la Tabla 5 con el ranking de prioridades de los criterios. El primer paso fue el cálculo de la aparición total de los criterios en los artículos (última fila de la Tabla 4) para definir el criterio con mayor prioridad, donde el criterio que apareció en más artículos fue el más prioritario (por ejemplo, el "precio" con 21). El problema aquí, es que a veces dos criterios tienen la misma aparición total de los criterios en los artículos, por ejemplo, la "capacidad técnica" y el "riesgo", con 7 como número total. Para solucionar esto, se han añadido dos valores más a la Tabla 4: la aparición total de los criterios por autor (última columna) y la prioridad total del artículo (penúltima fila).

La aparición total de los criterios por autor sólo suma el número de criterios de evaluación que un artículo definió y pone este valor en la última columna de la Tabla 4. Por ejemplo, el artículo número 3 de la Tabla 4 definió 3 criterios y este número fue colocado en la columna aparición total de los criterios por autor. La prioridad total del artículo suma el número de prioridad de los artículos ( $\mathrm{Pr}$, columna 2 de la Tabla 4).

Volviendo al ejemplo de los criterios de "capacidad técnica” y "riesgo", donde antes se indicó que tienen la misma aparición total de criterios en los artículos, y es imposible definir cuál tiene la mayor prioridad. En la Tabla 4, el criterio de "riesgo" apareció en los artículos 11, 18, 28, 30, 32, 41 y 52, con los respectivos números de prioridad 4, 7, 1, 8, 6, 3 y 4, y la "capacidad técnica" apareció en los artículos 8, 16, 20, 26, 29, 34 y 49, con los respectivos números de prioridad $6,3,4,4,5,5$ y 5 . Por lo tanto, la prioridad total del artículo fue 33 para el criterio "riesgo" y 32 para el criterio "capacidad técnica”. Como el número de prioridad menor es mejor, también la menor suma es mejor, por lo tanto una mayor prioridad fue dada al criterio de "capacidad técnica". Aquí es importante recordar que estos cálculos para la prioridad total del artículo, se utilizaron sólo para resolver el problema cuando dos criterios tienen una aparición total de los criterios en los artículos igual para la definición de las prioridades de los criterios de evaluación en la Tabla 5.

Por lo tanto, dos consideraciones deben ser hechas: 1) Los criterios precio, flexibilidad, calidad y entrega no tienen ningún cálculo de prioridad total del artículo debido a que no tienen el mismo número de aparición total de los criterios en los artículos con los demás criterios y 2) No es posible utilizar la prioridad total del artículo como primera forma de definir la prioridad de los criterios de evaluación. 
Tabla 5. El ranking actual y el anterior de los criterios de evaluación

\begin{tabular}{|c|c|c|c|c|c|}
\hline $\begin{array}{l}\text { Ranking } \\
\text { Actual }\end{array}$ & $\begin{array}{l}\text { Ranking } \\
\text { Anterior }\end{array}$ & Factor & $\begin{array}{l}\text { Ranking } \\
\text { Actual }\end{array}$ & $\begin{array}{l}\text { Ranking } \\
\text { Anterior }\end{array}$ & Factor \\
\hline 1 & 6 & Precio & 16 & 15 & $\begin{array}{l}\text { Soporte (servicio de } \\
\text { reparación) }\end{array}$ \\
\hline 2 & 1 & Calidad & No & 3 & Desempeño \\
\hline 3 & 2 & Entrega & No & 4 & $\begin{array}{l}\text { Garantías y Políticas } \\
\text { de Reclamación }\end{array}$ \\
\hline 4 & Nuevo & Flexibilidad & No & 5 & $\begin{array}{l}\text { Instalaciones de } \\
\text { Producción y } \\
\text { Capacidad }\end{array}$ \\
\hline 5 & 7 & Capacidad Técnica & No & 9 & $\begin{array}{l}\text { Cumplimiento de los } \\
\text { Procedimientos }\end{array}$ \\
\hline 6 & Nuevo & Riesgo & No & 10 & Comunicación \\
\hline 7 & 11 & $\begin{array}{l}\text { Reputación y Posición } \\
\text { en la Industria }\end{array}$ & No & 12 & Deseo de Negocios \\
\hline 8 & Nuevo & Compatibilidad & No & 14 & Control de Operación \\
\hline 9 & Nuevo & Tiempo & No & 16 & Actitud \\
\hline 10 & 8 & Posición Financiera & No & 17 & Impresión \\
\hline 11 & Nuevo & $\begin{array}{l}\text { Capacidad de } \\
\text { Respuesta }\end{array}$ & No & 18 & $\begin{array}{l}\text { Capacidad de } \\
\text { Embalaje }\end{array}$ \\
\hline 12 & 21 & $\begin{array}{l}\text { Experiencia (cantidad } \\
\text { de negocios } \\
\text { anteriores) }\end{array}$ & No & 19 & $\begin{array}{l}\text { Registro de Relación } \\
\text { Laboral }\end{array}$ \\
\hline 13 & Nuevo & Recursos Humanos & No & 20 & $\begin{array}{l}\text { Localización } \\
\text { Geográfica }\end{array}$ \\
\hline 14 & Nuevo & $\begin{array}{l}\text { Participación de } \\
\text { Mercado }\end{array}$ & No & 22 & $\begin{array}{l}\text { Asistencia a la } \\
\text { Formación }\end{array}$ \\
\hline 15 & 13 & $\begin{array}{l}\text { Administración y } \\
\text { Organización }\end{array}$ & No & 23 & $\begin{array}{l}\text { Acuerdos de } \\
\text { Reciprocidad }\end{array}$ \\
\hline
\end{tabular}

La Tabla 5 proporciona la clasificación actual así como la anterior de los diferentes criterios de evaluación. La clasificación actual (columna "Ranking Actual") se ha basado en los cálculos explicados anteriormente para definir el criterio con más prioridad. Primeramente, se ha utilizado la aparición total de los criterios en los artículos, como se ha explicado anteriormente, para definir el criterio con mayor prioridad. En los casos donde los criterios tenían una aparición total de los criterios en los artículos igual, se ha utilizado la prioridad total del artículo para el desempate. La columna "Ranking Anterior" se refiere a la clasificación que los criterios obtuvieron en el estudio de (Dickson, 1966). La Tabla 5 también incluye los nuevos criterios que no estaban presentes en la lista de 23 criterios.

\section{Conclusiones}

Esta revisión sistemática relacionada con la toma de decisión en la selección de proveedores de TI se ha llevado a cabo utilizando el protocolo propuesto por (Biolchini, et al., 2005). Este protocolo tiene cinco características principales relacionadas con el proceso de revisión sistemática, realizándose un análisis final estadístico de los estudios primarios para lograr resultados y también para comprobar la calidad de los trabajos seleccionados. La revisión sistemática lleva más tiempo y también más esfuerzo que la revisión de literatura normal, pero los resultados alcanzados son más consistentes, ya que se basan en un prototipo bien definido que detalla todos los métodos para la inclusión y exclusión de los estudios y de la información pertinente. 
El gran porcentaje (86\%) de los estudios analizados se relacionó con TI y SI, que son los principales temas que esta revisión sistemática se propuso analizar. En cuanto a los modelos utilizados y las propuestas definidas por los estudios, el modelo más utilizado fue el Analytic Hierarchy Process (AHP) con un 19\%, seguido por el modelo PROMETHEE con un 10\%. El 37\% de los estudios utilizaron una mezcla de dos o tres modelos de criterios múltiples. Estos resultados indican una falta de estudios en la toma de decisión de selección de proveedores de TI, ya que sólo 52 estudios primarios fueron encontrados y no todos ellos están específicamente relacionados con la definición de un modelo de decisión en la selección de proveedores de TI. La mayoría de los de los estudios (71\%), que están relacionados con el diseño de un modelo específico para la toma de decisiones, no desarrolló un modelo completo para ser utilizado en el área de TI, lo que es una motivación para el desarrollo de un trabajo futuro en relación con esta área.

Este estudio indica un cambio significativo de los diferentes criterios de evaluación y en su importancia relativa en comparación con la investigación realizada por (Dickson, 1966). Comenzando con el estudio de (Dickson, 1966) y utilizando los resultados de la revisión sistemática desarrollada por los autores de este artículo, se han definido 16 criterios para la selección de un proveedor de TI en base a la lista de 23 criterios definidos por (Dickson, 1966). Estos nuevos criterios de evaluación previamente definidos serán utilizados como parte de un modelo de decisión en la selección de proveedores de TI que será desarrollado por los autores de este artículo.

\section{Referencias bibliográficas}

Aarkstore. (2010). The Black Book Of Outsourcing: State Of The Outsourcing Industry 201O. Retrieved from http://www.articlesnatch.com/Article/The-Black-Book-OfOutsourcing--State-Of-The-Outsourcing-Industry-2010/1763430.

Aydin, M. N., \& Bakker, M. E. (2008). Analyzing IT Maintenance Outsourcing Decision From a Knowledge Management Perspective. Information Systems Frontiers, $10(3), 293-305$.

Basili, V., Craze, D., Jino, M., Mendonca, M., \& Shull, F. (2007). Extracting Information From Experimental Software Engineering Papers. Paper presented at the XXVI International Conference of the Chilean Society of Computer Science, $105-114$.

Biolchini, J., Mian, P., Natali, A., \& Travassos, G. (2005). Systematic Review in Software Engineering. Rio de Janeiro: COPPE/UFRJ.

Brans, J. P., Mareschall, B., \& Vincke, P. H. (1986). How to Select and How to Rank Projects: The PROMETHEE Method. European Journal of Operational Research, 14, 228-238.

Calvo-Manzano, J., Cuevas, G., Grossi, L., Mejia, J., \& San Feliu, T. (2009). Outsourcing Contracts: A Systematic Review. International Book Series "INFORMATION SCIENCE \& COMPUTING", 11, 107-115. 
Calvo Manzano, J. A., Cuevas, G., Gasca, G., \& San Feliu, T. (2009). State of the art for risk management in software acquisition. SIGSOFT Softw. Eng. Notes, 34(4), 110 .

CMMI, P. T. (2010). CMMI for Acquisition, Version 1.3 (CMU/SEI-2010-TR-032): Software Engineering Institute.

Corbet, \& Associates. (2002). Outsourcing's Next Wave.

Dialani, M. (2010). Worldwide and U.S. Research and Development/Product Engineering Services 2010-2014 Forecast: Will the Economic Downturn and Changing Customer Needs Enable Increased Outsourcing of These Services? Retrieved from http://www.idc.com/research/viewdocsynopsis.jsp?containerId=225954\&section $\underline{\mathrm{Id}=\text { null\&elementId }=\text { null\&pageType }=\text { SYNOPSIS. }}$.

Dickson, W. G. (1966). An Analysis of Vendor Selection Systems and Decisions. Journal of Purchasing, 2, 5-20.

Dorothy, L., \& Dwayne, W. (2006). Bringing IT Back: An Analysis of The Decision to Backsource or Switch Vendors. Decision Sciences, 37(4), 605-621.

El-Emam, K., Hoaglin, D. C., Jones, P. W., Kitchenham, B. A., Pfleeger, S. L., Pickard, L. M., et al. (2001). Preliminary Guidelines For Emprical Research in Software Enginerring. National Research Council of Canada.

Garcia, F., Piattini, M., \& Pino, F. (2008). Software Process Improvement in Small and Medium Software Enterprises: A Systematic Review. Software Quality Journal, 16(2), 237-261.

Gellings, C. (2007). Outsourcing Relationships: The Contract as IT Governance Tool. Paper presented at the 4oth Annual Hawaii International Conference on System Sciences, 236c-236c.

Gill, S., Grewal, C. S., \& Sareen, K. K. (2008). A Multicriteria Logistics-Outsourcing Decision Making Using The Analytic Hierarchy Process. Int. J. Services Technology and Management, 9(1), 1-13.

Huai, J. (2007). An Incentive Model of IS Outsourcing Contract. Paper presented at the International Conference on Wireless Communications, Networking and Mobile Computing, 6588-6592.

Lee, A. H. I. (2009). A Fuzzy Supplier Selection Model With The Consideration of Benefits, Opportunities, Costs and Risks. Expert Syst. Appl., 36(2), 2879-2893.

Mikhailov, L., \& Singh, M. G. (2003). Fuzzy analytic network process and its application to the development of decision support systems. IEEE Transactions on Systems Man and Cybernetics Part C Applications and Reviews, 33(1), 33-41.

Saaty, T. L. (1980). The Analytic Hierarchy Process. New York: Mc Graw-Hill. 